Y.-H. Chu, N.B. Suntzeff, J.E. Hesser, and D.A. Bohlender, eds.

\title{
Molecular Line Observations in the Magellanic Clouds
}

\author{
Lars E.B. Johansson, Arto Heikkilä \\ Onsala Space Observatory, S-439 92 Onsala, Sweden
}

\author{
Hans Olofsson \\ Stockholm Observatory, S-133 36 Saltsjöbaden, Sweden
}

\begin{abstract}
We have observed spectral line emission from a sample of clouds in the Magellanic Clouds to investigate the effects of metallicity and FUV radiation on the physical and chemical properties of the interstellar medium. The clouds were identified by $\mathrm{CO}$ surveys and selected to cover a wide range of environments. We present molecular line data for five clouds in the LMC and one cloud in the SMC.
\end{abstract}

\section{Introduction}

The Magellanic Clouds provide us with excellent laboratories for studies of the effects of metallicity variations on the chemical and physical properties of the interstellar medium. Here we present observational results from the Swedish-ESO Submillimetre Telescope (SEST) which allows studies of individual molecular clouds at scales smaller than $5 \mathrm{pc}$, i.e. well below the sizes of the largest molecular cloud complexes in the Galaxy. We present molecular line data for a sample of clouds, identified by CO surveys. The LMC sample (30Dor-10, 30Dor-27, N160, N159W, N159S; see Johansson et al. 1998) is located in an area including the 30 Doradus nebula and its southern HII regions N160 and N159. One cloud in the SMC bar, N27 (also denoted LIRS 49), is included in the sample.

\section{CO in the Magellanic Clouds}

The CO surveys (see e.g. Johansson et al. 1998 and references therein) have shown that the bulk of the $\mathrm{CO}$ emission originates in clouds or cloud complexes of sizes no larger than about $20 \mathrm{pc}$, surrounded by large areas apparently devoid of $\mathrm{CO}$ gas. Thus, the surface filling factor of the $\mathrm{CO}$ emission is small on size-scales of a few $100 \mathrm{pc}$. Low CO filling factors, relative to the Galaxy, seem to apply to the clouds as well. This is indicated by radiative transfer calculations using $\mathrm{CO}$ isotopomers observed in the three lowest rotational transitions typical volume filling factors of the $\mathrm{CO}$ gas are less than $10^{-1}$. These properties suggest that photon-dominated regions occupy a larger fraction of the ISM in the Magellanic Clouds than in the Galaxy, a result of the reduced dust abundances and, accordingly, a deeper penetration of the UV radiation into the ISM. 


\section{Molecular Abundances}

The number of detected interstellar molecules is at present 16 (26 including isotopomers) in the LMC and 10 (14) in the SMC. The most recent detections are those of hydrogen sulphide $\left(\mathrm{H}_{2} \mathrm{~S}\right)$ and methyl acetylene $\left(\mathrm{CH}_{3} \mathrm{CCH}\right)$ in $\mathrm{N} 159 \mathrm{~W}$ in the $\mathrm{LMC}$ and $\mathrm{H}^{13} \mathrm{CO}^{+}$in $\mathrm{N} 27$ in the SMC (Heikkilä et al. 1999). In addition, there are a few tentative detections, e.g. $\mathrm{C}^{34} \mathrm{~S}$ in the SMC (Chin et al. 1998) and $\mathrm{H}_{2} \mathrm{CS}$ in the LMC (Heikkilä et al. 1999).

To derive fractional abundances we have estimated the $\mathrm{H}_{2}$ contents using the virial mass concept. Typical abundances are $5 \times 10^{-10}$ and $1 \times 10^{-10}$ in the LMC and the SMC, respectively. After the $\mathrm{CO}$ isotopomers, the ethynyl radical $\left(\mathrm{C}_{2} \mathrm{H}\right)$ is the most abundant trace molecule observed.

Relative to Galactic clouds, the derived abundances in N159W are typically 5 to 20 times lower. Two of the clouds, N27 in the SMC and 30Dor-10 in the central parts of the 30 Doradus nebula show significantly lower abundances than the rest of the sample; on the average a factor of 7 relative to those in N159W. In $\mathrm{N} 27$, the most likely explanation is the lower metallicity in the SMC while the underabundances in 30Dor-10 probably are due to higher photodissociation rates caused by the intense FUV radiation field in this area.

\section{Physical and Chemical Properties of the Gas}

The kinetic temperatures derived mirror the environment of the clouds: regions associated with vigorous star formation activity are warmer. A comparison between number densities and average densities, calculated from the virial masses, indicates that the clouds have a very clumpy structure. The number density and kinetic temperature estimates are generally based on an excitation analysis of $\mathrm{CO}, \mathrm{CS}, \mathrm{SO}, \mathrm{HCO}^{+}$and $\mathrm{H}_{2} \mathrm{CO}$.

Relative to the Galaxy, the Magellanic Clouds seem to be characterised by a higher degree of photon-driven chemistry. This is indicated by the relatively high abundances of $\mathrm{C}_{2} \mathrm{H}$, but also as abundance variations of $\mathrm{C}_{2} \mathrm{H}$ and $\mathrm{HCO}^{+}$ with the environment. The formation of both molecules have reaction routes involving $\mathrm{C}^{+}$. Our data suggest that more complex molecules do not show significantly smaller abundances than simpler species in the ISM of the Magellanic Clouds. For example, the methanol abundance in $\mathrm{N} 159 \mathrm{~W}$ is similar to that of less complex molecules.

\section{References}

Chin, Y.-N., Henkel, C., Millar, T.J., et al. 1998, A\&A, 330, 901

Heikkilä, A., Johansson, L.E.B., \& Olofsson, H. 1999, A\&A, 344, 817

Johansson, L.E.B., Greve, A., Booth, R.S., et al. 1998, A\&A, 331, 857 